\title{
Stochastic dynamics of leukemic cells under an intermittent targeted therapy
}

\author{
Nicola Pizzolato • Dominique Persano Adorno • \\ Davide Valenti · Bernardo Spagnolo
}

Received: 18 November 2009/ Accepted: 14 February 2011

(C) Springer-Verlag 2011

\begin{abstract}
The evolutionary dynamics of cancerous cell populations in a model of Chronic Myeloid Leukemia (CML) is investigated in the presence of an intermittent targeted therapy. Cancer development and progression is modeled by simulating the stochastic evolution of initially healthy cells which can experience genetic mutations and modify their reproductive behavior, becoming leukemic clones. Front line therapy for the treatment of patients affected by CML is based on the administration of tyrosine kinase inhibitors, namely imatinib (Gleevec) or, more recently, dasatinib or nilotinib. Despite the fact that they represent the first example of a successful molecular targeted therapy, the development of resistance to these drugs is observed in a proportion of patients, especially those in advanced stages. In this study, we simulate an imatinib-like treatment of CML by modifying the fitness and the death rate of cancerous cells and describe the several scenarios in the evolutionary dynamics of white blood cells as a consequence of the efficacy of the different modeled therapies. The patient response to the therapy is investigated by simulating a drug administration following a continuous or pulsed time scheduling. A permanent disappearance of leukemic clones is achieved with a continuous therapy. This theoretical behavior is in a good agreement with that observed in previous clinical investigations. However, these findings demonstrate that an intermittent therapy could represent a valid alternative in patients with high risk
\end{abstract}

N. Pizzolato $(\bowtie) \cdot D$. Persano Adorno · D. Valenti .

B. Spagnolo

Dipartimento di Fisica, Group of Interdisciplinary Physics,

Università di Palermo, Viale delle Scienze, ed. 18,

90128 Palermo, Italy

e-mail: npizzolato@gip.dft.unipa.it

URL: http://gip.dft.unipa.it of toxicity. A suitable tuned pulsed therapy can also reduce the probability of developing resistance.

Keywords Stochastic dynamics - Cancer evolution . Complex systems

\section{Introduction}

Leukemia is a form of cancer of the blood cells. Healthy white blood cells grow and divide in an orderly and controlled way, but in leukemia the process gets out of control and the cells divide too quickly. In Chronic Myeloid Leukemia (CML), too many myeloid cells (one of the main types of white blood cells) are produced and released into the blood when they are immature and unable to work properly, leading to an increased risk of infection and strongly limiting the production of healthy red cells and platelets. A specific chromosomal abnormality, named Philadelphia $(\mathrm{Ph})$ chromosome, is associated with the development of CML. It consists in a reciprocal translocation occurring in stem cells and progenitors (Michor 2007); part of the BCR ("breakpoint cluster region") gene from chromosome 22 is fused with part of the ABL gene on chromosome 9 (ABL stands for "Abelson," the name of a leukemia virus which carries a similar protein). BCR-ABL fusion gene, which is located on the shorter chromosome 22 , activates a number of cell cycle controlling proteins and tyrosine kinase enzymes, speeding up cell division and causing genomic instability.

Front line therapy in CML is represented by tyrosine kinase inhibitors that turn off the signal produced by the $\mathrm{Ph}$ chromosome, limiting the proliferation of BCR-ABL expressing hematopoietic cells. These inhibitors greatly reduce the growth of the tumor clone but can cause toxicity 
on full-treated patients (Faber et al. 2006). Moreover, immature leukemic cells are affected by a chromosomal instability that brings to a great number of genetic alterations. In particular, point mutations within the protein tyrosine kinase domain in the BCR-ABL gene itself (Barthe et al. 2001; Weisberg and Griffin 2001, 2003) are the basis for the development of acquired resistance to imatinib-like drugs in a relevant fraction of patients.

A new therapeutic approach, based on the intermittent dosage of tyrosine kinase inhibitors, has been recently investigated on clinical studies devoted to the reduction of hematologic toxicity in patients affected by CML (Faber et al. 2006; Shah et al. 2008; Martinelli et al. 2009). These studies have demonstrated that an intermittent therapy (IT) can be considered a good alternative to the standard daily dosing in patients with persistent signs of myelotoxicity, without compromising the patient cytogenetic response. Recent clinical studies on the treatment of hormone-sensitive advanced prostate cancer have demonstrated that the introduction of breaks in long-term anticancer treatments can prolong tumor response to the therapy (Seruga and Tannock 2008; André and Pasquier 2009). Similar results are also reported by Sabnis et al. (2008) on a breast cancer clinical study. A "drug-driven dependency deprivation effect" might be able to inhibit and destroy the most resistant or dependent cancer clones. In CML context, a temporary interruption of imatinib therapy was found to significantly reduce the presence of leukemic cells showing the BCR-ABL Y252H (P-loop) mutation in a resistant patient (Müller et al. 2002; Hochhaus and La Rosée 2004).

Following these ideas, we like to achieve two goals by modeling, first reducing drug toxicity during a continuous imatinib-based treatment, second designing a strategy to stave off resistance. We have designed a model of leukemic cell evolutionary dynamics for a statistical study of cancer development in patients treated by an imatinib-like IT. The aim of this study is to find the optimal therapeutic approach that minimize imatinib side effects, maximizing the clinical benefits of this treatment and the quality of life of the patient. In "Model of CML evolution" section, we describe the model and give the details of the simulation process. Theoretical results and a comparison with clinical investigations are reported in "Results and discussion" section. Conclusions are drawn in "Conclusions" section.

\section{Model of CML evolution}

The evolutionary dynamics of cancer initiation and progression has been theoretically approached with mathematical deterministic equations (Michor et al. 2005, 2006;
Abbott and Michor 2006; Garner et al. 2006) or with stochastic models (Roeder et al. 2006; Brumer et al. 2006; Iwasa et al. 2004; Michor et al. 2003; Dingli and Michor 2006; Komarova and Wodarz 2007; Zhdanov 2008; Pizzolato et al. 2009), both using the basic idea that cancer arises when a single non-differentiated cell experiences multiple mutations, as confirmed by numerous studies on cancer genetics (Knudson 2001; Frank et al. 2003). These studies describe the temporal evolution of the level of BCR-ABL positive cells, observed in clinical investigations on patients treated with imatinib, in terms of a partial or total failure of the drug efficacy on cancer cells.

We study the evolutionary dynamics of $N=10^{4}$ replicating cells. This value is several orders of magnitude lower than the typical total contents of blood cells in humans, but it is great enough for the statistical study of the cancer development in a single blood compartment. The total number of cells is kept constant during the time evolution, as it can be reasonably assumed for a blood cancer. In order to simulate the random process of cell selection for reproduction, mutation, and death, we adopt a Monte Carlo approach, as already done by several authors in theoretical cancer studies (see Iwasa et al. 2004; Michor et al. 2006; Zhdanov 2008; Pizzolato et al. 2009).

In the proposed model, cells are distributed over four populations: healthy cells (type-0), first-mutated cells (type-1), double-mutated leukemic cells (type-2), and resistant leukemic clones (type-3). In a real biological context, each population of blood cells is composed of the sum of four sub-populations: stem cells, progenitors, differentiated, and terminally differentiated cells. Stem cells give rise to progenitors, which produce differentiated cells, which produce terminally differentiated cells. This hierarchy applies both to normal and leukemic cells. Because the blood predominantly contains terminally differentiated cells, clinical values give an estimate of the fraction of terminally differentiated leukemic cells (Michor et al. 2005). Therefore, in view of devising a model which accounts for clinical findings, we consider only the dynamics of differentiated cells. In this context, the absence, at a certain time, of differentiated cells belonging to a given population, e.g., healthy or first-mutated cells, does not imply their absence at successive times. In fact, type- 0 or type- 1 cells can be produced by stem cells of the same type, which are always present in blood, even if in low percentage.

In the simulations, where time is measured in units of cell divisions, we assume that each single time step is long enough to allow the stem cells to become a differentiated or a terminally differentiated cells. In the model, we take into account the presence of stem cells and progenitors only for what concerns the choice of mutation rates and fitness of the four simulated populations. In fact, since 
differentiated and terminally differentiated cells can arise by stem cells, which are characterized by an indefinite potential for self-renewal (Michor et al. 2005), or by progenitors (Michor 2007), mutation rates and fitness are considered in the model as averages over these two sub-populations only. Conversely, death rates can be considered as averages over the values of all four subpopulations.

We have scaled the simulation time from cell divisions to days by using a simulation time step of 0.002 days, corresponding to 500 cell divisions in one day. This scaling has been chosen in order to achieve a complete restore of healthy cells in almost 100 days, as observed in clinical cases of optimal therapy response (Michor et al. 2005; Roeder et al. 2006).

The stochastic dynamics of the cancer evolution is modeled by assuming that cells reproduce asynchronously. This means that each elementary step of the stochastic process consists of a birth and a death event, i.e., a Moran process (Moran 1962). The exact sequence of logical steps for a single reproduction and death event is described by the flowcharts shown in Figs. 1 and 2, respectively. For the birth, one of the $N$ cells is randomly chosen proportionally to its capacity to reproduce. The fitness of type- 0 and type1 cells are set equal to 1 as already adopted in other theoretical studies (Iwasa et al. 2004; Pizzolato et al. 2009). In the absence of therapy, the reproductive rate of a leukemic cell is assumed to be ten times that of a normal cell. This value is suitable to describe the stochastic dynamics of the leukemic cells after the disease has reached a "macroscopic" stage of evolution, while the dormant phase of CML, characterized by a latency time of about 5-7 years (Ichimaru et al. 1981; Horn et al. 2008), should be investigated by using in the model lower fitness values of the leukemic cells.

The imatinib-based treatment lowers this value only for those cells which are sensitive to the drug; resistant leukemic cells are unaffected by the therapy and their reproductive capability remains unchanged. In particular, we have assumed that imatinib reduces the fitness of type- 2 leukemic cells to 0.04 . Fitness values have been chosen in order to match the response of type-2 leukemic cells to that observed in patients treated by an imatinib-based targeted therapy (Michor et al. 2005; Roeder et al. 2006).

Healthy cells mutate to cells of type-1 (first allele mutation) at a rate $M_{01}$ equal to 0.0005 ; type- 1 cells mutate to type-2, which are leukemic cells sensitive to the therapy, at a rate $M_{12}$ equal to 0.002 . These values, comparable with the mutation rates adopted in the models of Refs. Iwasa et al. (2004); Michor et al. (2005), guarantee a good agreement between these findings and clinical results. We performed simulations also for different values of $M_{01}$ and $M_{12}$, finding that only the waiting time before the first appearance of a leukemic cell is sensitive to these values
Fig. 1 Flowchart of the reproduction event in a single time step. $\mathrm{F}_{N i}$ (with $i=0,1,2,3$ ) are the normalized fitness rates; $M_{01}, M_{12}, M_{23}$ are mutation rates for transitions from type- 0 to type- 1 cells, from type- 1 to type- 2 cells, and from type- 2 to type- 3 cells, respectively

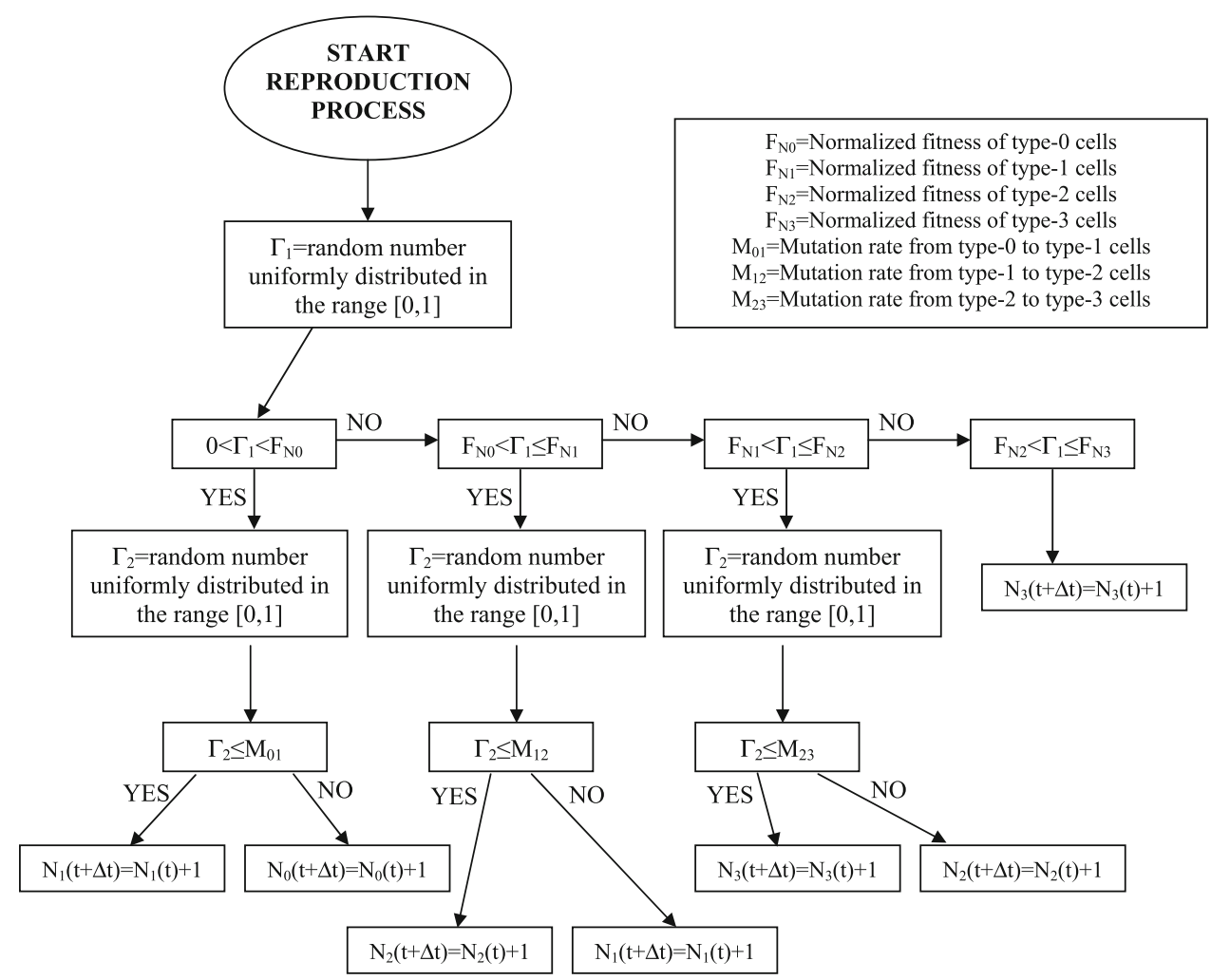


Fig. 2 Flowchart of the death event in a single time step. $\mathrm{D}_{N i}$ (with $i=0,1,2,3$ ) are the normalized death rates

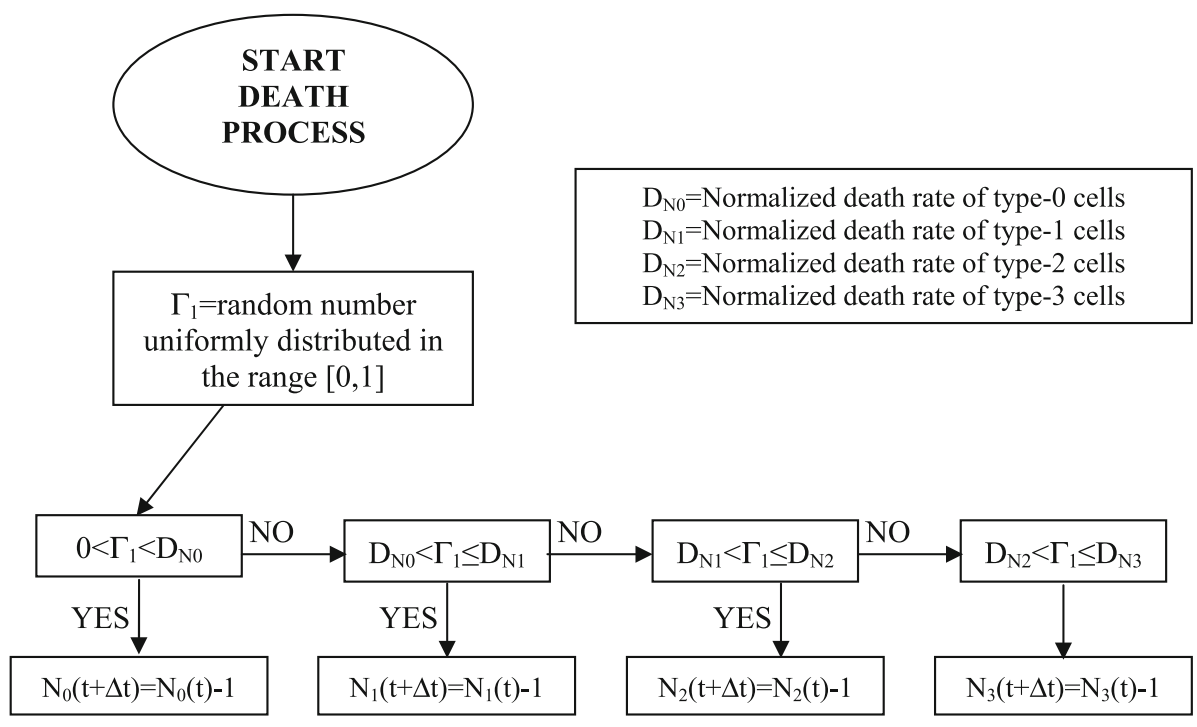

and they do not significantly affect the subsequent course of the disease. Since the tyrosine kinase inhibition by imatinib induces apoptosis in BCR-ABL expressing cells (Vigneri and Wang 2001; Belloc et al. 2007), we take into account this effect by increasing the death rate of type-2 leukemic cells with respect to the other cell populations during the therapy. We do not consider back mutations and neglect direct transitions from healthy (type-0) to leukemic cells (type-2) or to resistant clones (type-3).

For the study of real cases of therapy effects on cancer evolution, we have supposed that the cell system could react to the drug administration by activating multiple genetic changes that cause an enhancement of the mutation rates from normal to cancerous cells. This assumption is supported by the clinical evidence that certain mutations increase the rate at which subsequent mutations occur (Jackson and Loeb 1998; Loeb et al. 2002). Clinical studies show an increasing trend in the number of patients developing resistance to imatinib within two years of treatment, with the progression of the disease through the chronic, accelerated and blast crisis phases (Hochhaus and La Rosée 2004). These phases are characterized by the presence of an increasing number of immature leukemic cells (myeloblasts) in the blood or bone marrow (Sokal et al. 1988). Therefore, we have assumed in the model that type- 2 cells become resistant type- 3 by mutating at a rate $M_{23}$ which has not a constant value, but increases with the number $\mathrm{N}_{2}$ of leukemic double-mutated cells (Fig. 3). The theoretical trend of $M_{23}$ versus $N_{2}$ has been extrapolated from the behavior of the frequency of acquired hematologic resistance as a function of the number of myeloblasts in the blood, accordingly with the standard definition of CML phases cited above (Hochhaus and La Rosée 2004), and calculated by the equation

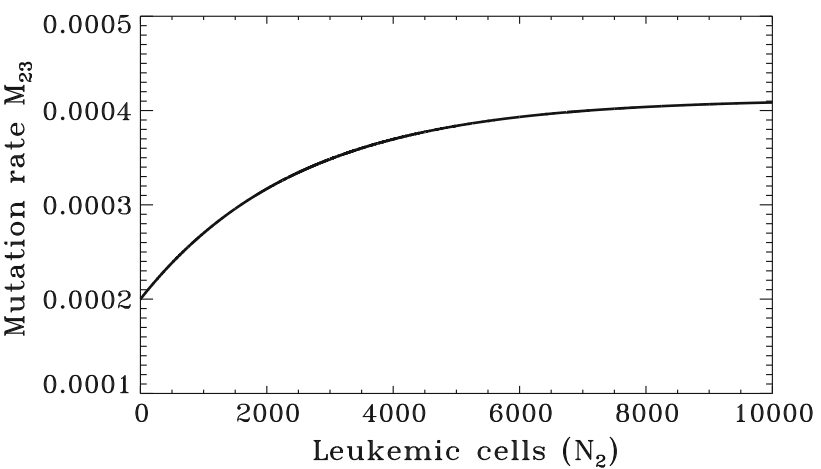

Fig. 3 Mutation rate $M_{23}$ as a function of the number of leukemic cells sensitive to the therapy, calculated by Eq. $1 . M_{23}$ reproduces the behavior of the frequency of deleterious mutations associated to the appearance of resistance as a function of disease progression, observed in clinical investigations Hochhaus and La Rosée (2004)

$M_{23}=a+\left(b \cdot\left(1-\mathrm{e}^{-N_{2} / c}\right) / d\right)$

where $a=2 \times 10^{-4}, b=8.5 \times 10^{3}, c=2.5 \times 10^{3}$, and $d=4 \times 10^{7}$ are the best parameters for which the Eq. 1 reproduces the behavior observed in clinical investigations (Hochhaus and La Rosée 2004). The mutation rate $M_{23}$ has a latency value of $2 \times 10^{-4}$, and it progressively reaches the double of its value in the presence of an increase of immature cells $\mathrm{N}_{2}$.

Since the specific chromosomal mutation (or set of mutations) that causes the cancerous cell to become resistant and insensitive to the therapy can be considered an evolutionary reaction of the cell against the drug, we assume that, during the periods of absence of therapy, the mutation rate $M_{23}$ is reduced to such very low values that we reasonable keep it equal to zero. 


\section{Results and discussion}

In this study, we investigate the response to imatinib-based therapies, characterized by different drug administration times, in patients where the proportion of malignant cells has reached almost $99 \%$ at the time of diagnosis. Hence, the initial condition in the simulations is that all the patient differentiated cells are of leukemic type-2. As discussed in the previous section, healthy and first-mutated cells participate to the reproduction process, although their initial value is set to zero, because stem cells of these two populations, even if not directly included into the counting of cells, are always able to generate a new healthy or a first-mutated cell. In this context, the resistant clone may originate only by a stochastic process of mutation from the leukemic phenotype. Type- 3 cells acquire the chance to reproduce themselves only after the first resistant cell is generated.

The standard daily dosage of imatinib for CML patients is $400 \mathrm{mg} / \mathrm{day}$ (Rea et al. 2009). Because this value is sufficiently high to persist in patient plasma for about $24 \mathrm{~h}$ (Faber et al. 2006), the standard treatment of CML is achieved with a continuous therapy. In order to lower the number of leukemic cells as rapidly as possible during the first 100 days of treatment, patients are simulated to be treated with imatinib with the standard daily dosage, corresponding to a fully continuous modeled therapy.

This choice has been made first to support patient's health, and second to reduce the probability of developing resistance. Resistance is greater when the number of immature leukemic cells is higher. For the subsequent 300 days, we have simulated three type of pulsed therapies, with the standard dose administration interrupted by a time break of 0.4 day $(\sim 10 \mathrm{~h}), 0.6$ day $(\sim 14 \mathrm{~h})$, and 0.7 day $(\sim 17 \mathrm{~h})$. The evolution of the same system of cells is shown, for comparison, in the presence of a continuous treatment for all 400 days.

Individual patient response under continuous therapy

The evolutionary dynamics of healthy and cancerous cells are first investigated by monitoring the individual patient response to a continuous therapy, focusing the attention on the development of resistance. In Fig. 4, we show three representative cases of patient response in the presence of acquired resistance. The data points are all sampled every 30 days starting from $t=10$ days. The upper panel describes a patient which initially responds to the therapy but, unfortunately, develops resistance in the very early phase of the treatment, even before the complete restoration of healthy cells. In the central and lower panels, we show the cell evolution of a patient which reaches a complete response to the therapy almost eradicating the leukemic phenotype (solid red line). However, since the
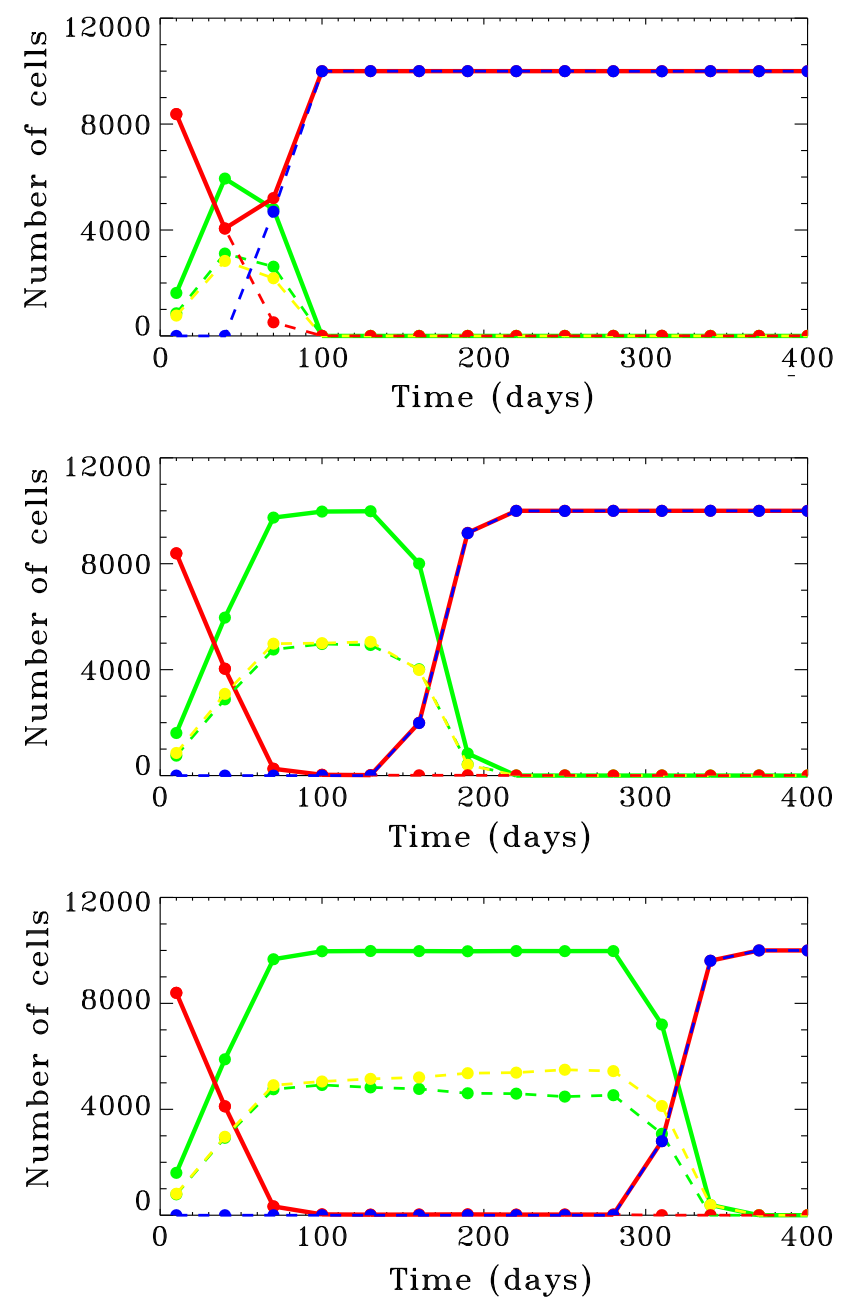

Fig. 4 Temporal evolution of cell populations for three representative patients developing resistance under continuous therapy. Healthy cells (dashed green line), first-mutated cells (dashed yellow line), leukemic cells still sensitive to the therapy (dashed red line), and resistant leukemic clones (dashed blue line). Continuous green line is referred to the sum of healthy and type- 1 cells and a continuous red line is used for the sum of type- 2 and resistant cells. (Color figure online)

number of leukemic type- 2 cells is still greater than zero, after same time delay, a mutation event leads to the birth of a resistant clone that briefly brings the system of cells to relapse.

The functional behavior of the number of leukemic cells with time (solid red lines in Fig. 4), decreasing during the efficacy phase of the therapy and subsequently increasing because of the appearance of resistance, has been compared with the clinical trends reported by Michor et al. (2005). In particular, we consider the case of a continuous drug administration therapy and compare, in Fig. 5, the results of the simulations, i.e., the percentage of leukemic cells, with the BCR-ABL values as a percentage of the BCR transcript levels observed in clinical investigations and reported in 


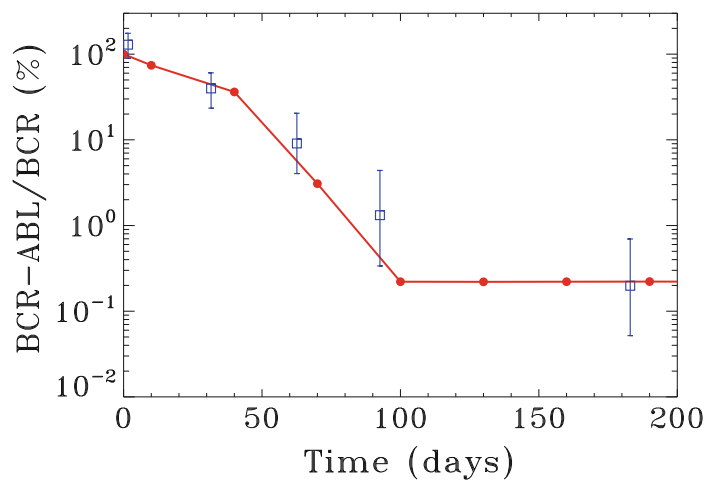

Fig. 5 Temporal evolution of the percentage of leukemic cells for the first 200 days of treatment characterized by a continuous drug administration. Red dots connected by the red line represent the percentage of modeled cancerous cells averaged over all those simulated patients who did not develop resistance during the first year of therapy. For comparison, we also plot the median with quartiles (blue squares and bars) of the $\mathrm{BCR}-\mathrm{ABL}$ values expressed as a percentage of the BCR transcript levels taken over real patients who do not have a rise in the leukemic cell burden during the first 12 months of therapy (data published in Michor et al. (2005)). (Color figure online)

Michor et al. (2005). Specifically, the simulated data decrease with a trend which is in a good agreement with the time behavior of the BCR-ABL/BCR percentage observed in clinical data, with a reduction of both simulated and clinical percentages around $1 \%$ within the first 100 days of therapy. This finding validates the model and justifies the choice of treating the simulated patients by a continuous drug administration therapy during the first 100 days.

Resistance start up during IT and average cell evolution

The occurrence of a genetic alteration that leads a leukemic cell to become resistant to the therapy is a stochastic process. Hence, in order to give a statistical significance to the descriptions, we have repeated every simulation $5 \times 10^{3}$ times.

First of all, we have estimated the mean waiting time for the development of resistance and the average increasing trend in the number of resistant cells. The mean waiting time before the first appearance of a resistant clone has been calculated by averaging over all simulated patients that have developed resistance within 400 days. However, the number $n_{\mathrm{c}}$ of resistant patients under continuous therapy is greater than the number $m_{i}$ (the index $i$ refers to different ITs) observed during the three cases of IT. Hence, we have normalized the samples of resistant patients under IT by assuming that among all patients which have not developed resistance under intermittent treatment, $n_{\mathrm{c}}-m_{i}$ will acquire resistance at the 400th day. In this way, the mean waiting time is calculated by averaging over the same number of patients. For every therapeutic approach, the average

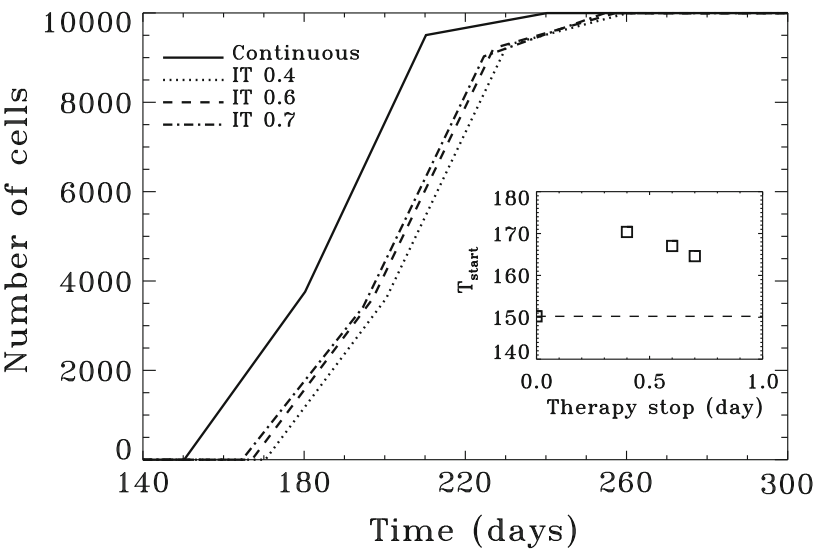

Fig. 6 Average trend of resistance development under continuous therapy (solid line) and IT with breaks of 0.4, 0.6, and 0.7 days (dotted, dashed, and dotted-dashed lines). The mean waiting time before the first appearance of a resistant type- 3 cell is calculated by averaging over all patients developing resistance. The average increasing trend of resistant cells is determined by scaling all the resistant patient trends with respect to the mean waiting time and calculating the average number of type- 3 cells on equivalent times. The inset shows the mean waiting time for patients developing resistance under the three modeled ITs, characterized by different therapy interruptions, with respect to that expected under continuous therapy

increasing trend of resistant cells with time has been determined by firstly shifting all resistant patient trends in a way that each single starting point coincided with the mean waiting time and, after that, we have calculated the average number of type- 3 cells on equivalent times.

In Fig. 6 we show that, by adopting an IT instead of the continuous one, an evident time delay into the appearance of resistance can be obtained. In particular, the best result in terms of a longer waiting time is achieved with the standard daily dosage alternative with a break of 0.4 day (see the inset of Fig. 6). Except for the starting time of resistance, the average progression trend of resistant cells does not show significant differences between the cases of IT and that of continuous therapy.

In Fig. 7, we show the temporal evolution of the number of healthy and first-mutated cells (green lines), cancerous cells still sensitive to the therapy (red lines) and resistant clones (blue lines), averaged over the whole set of simulated patients. A full continuous therapy throughout all 400 days is described by solid lines. The evolution of the same system of cells is shown, after 100 days of continuous treatment, in the presence of an IT characterized by the same dose duration and three different values of breaks.

Of course the best results, in terms of a permanent disappearance of the leukemic type- 2 cells, is achieved with a continuous therapy. However, the results shown in Fig. 7 demonstrate that an IT could also represent a valid choice in patients with high risk of toxicity. In fact, even if an increase of the average number of leukemic cells sensitive 


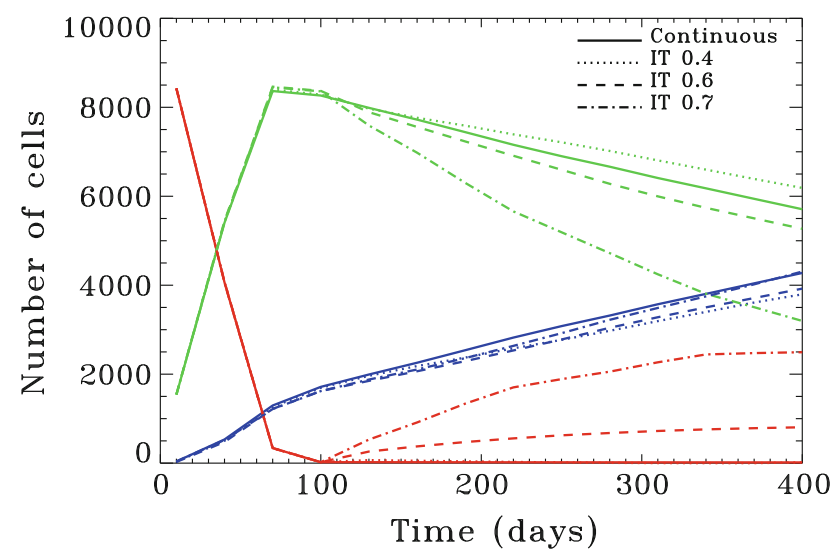

Fig. 7 Temporal evolution of cell populations: Healthy and firstmutated cells (green lines), leukemic cells still sensitive to the therapy (red lines), and resistant leukemic clones (blue lines). These values of the number of cells represent averages over 5000 simulated patients. Continuous lines are referred to the continuous therapy. Dotted, dashed, and dotted-dashed lines show the dynamics of the system of cells in the presence of an IT with breaks of $0.4,0.6$, and 0.7 days, respectively. The dotted red line is almost coincident with the red continuous one. (Color figure online)

to the therapy (red lines) is observed during an IT, this effect is partially counterbalanced by a reduction on the average number of resistant cells. In the best case, the IT with breaks of 0.4 days reduces the average number of resistant cells without causing a significant increase on the number of leukemic type- 2 cells (in Fig. 7 the dotted red line is almost coincident with the continuous one). When larger time windows between two consecutive doses become necessary, the average number of cancerous cells starts to increase again, suggesting a change of therapeutic strategy.

The observed reduction on the average number of resistant cells during IT with breaks of 0.4 days is essentially due to a lower number of patients developing resistance. In Fig. 8 we show the percentage of patients

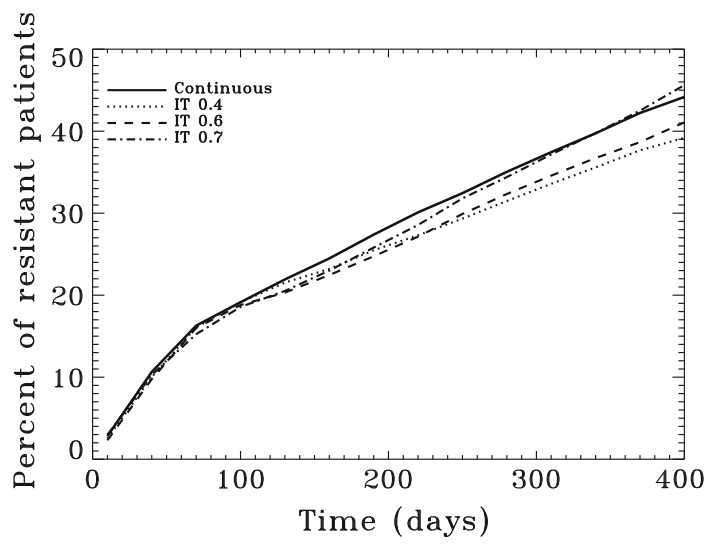

Fig. 8 Percentage of patients developing resistance as a function of time. Solid line represents the case of a continuous therapy. Dotted, dashed, and dotted-dashed lines represent an IT with breaks of 0.4, 0.6 , and 0.7 days, respectively developing resistance as a function of time, following the aforementioned therapeutic strategies. The statistical error associated to these values, calculated by repeating the set of simulations for 10 times, is less than $0.5 \%$. We find an advantage of the IT with breaks of 0.4 days over the continuous therapy approach for the whole duration of 400 days. IT characterized by therapy breaks of 0.6 days shows a comparable improvement in terms of a reduction in the percentage of patients developing resistance, while IT with breaks of 0.7 days, after an initial positive response, shows a worsening, becoming essentially equivalent to the continuous case.

\section{Conclusions}

Recent clinical oncology investigations indicate that an IT reduces both the incidence of toxicity and the complications that arise from the side effects due to a long-term continuous treatment with imatinib. In this study, by using a computational model, we have compared the effects of an intermittent imatinib-based therapy on the evolutionary dynamics of normal and cancerous cells in patients affected by CML, with respect to the standard treatment achieved by the continuous drug administration. Specifically, we find a good agreement between the theoretical results (Fig. 5) and those obtained in clinical investigations (Michor et al. 2005). With the aim of finding a less aggressive but still effective therapy, we have simulated the evolutionary dynamics of healthy and leukemic cells of 5000 patients in the presence of three type of pulsed therapies with the standard daily dose administration delayed by different time lags.

We have found that an IT can represent a valid alternative in patients with high risk of toxicity, without compromising the chances of a sustained tumor suppression. For therapy breaks until 0.6 day, the presence of a slight increase on the average number of leukemic cells is partially counterbalanced by a reduction on the average number of resistant clones. Furthermore, we have shown that an IT with a daily dosage retarded by a break of 0.4 day results into a more effective alternative with respect to a continuous therapy.

An IT with a break of 0.4 day reduces the percentage of patients developing resistance to the drug after 400 day of treatment of about $5 \%$. Hence, the introduction of breaks in anti-cancer therapy could prevent the treatment failure due to the developing of drug resistance. At last, but not least, all ITs show an appreciable time delay in the resistance switching on, with respect to the case of a continuous therapy.

Going behind the numerical details of the simulations, these findings show that an IT, appropriately tuned on 
specific patient necessities, should be preferred to the continuous one. The introduction of short breaks in longterm imatinib-based therapies can potentially enhance the treatment efficacy and considerably improve the quality of life of CML patients.

Acknowledgements This study was partially supported by MIUR. N. P. wish to thank Prof. Clementina Caracciolo and Dr. Rita Barone of the Department of Hematology and Bone Marrow Transplant Unit, Palermo-Italy, for helpful discussions.

\section{References}

Abbott LH, Michor F (2006) Mathematical models of targeted cancer therapy. Br J Cancer 95:1136-1141

André N, Pasquier E (2009) Response to "intermittent androgen blockade should be regarded as standard therapy in prostate cancer". Nat Clin Pract Oncol 6:E1

Barthe C, Cony-Makhoul P, Melo JV, Reiffers J, Mahon FX (2001) Roots of clinical resistance to STI-571 cancer therapy. Science 293:2163a

Brumer Y, Michor F, Shakhnovich EI (2006) Genetic instability and the quasispecies model. J Theor Biol 241:216-222

Belloc F, Moreau-Gaudry F, Uhalde M, Cazalis L, Jeanneteau M, Lacombe F, Praloran V, Mahon FX (2007) Imatinib and nilotinib induce apoptosis of chronic myeloid leukemia cells through a bim-dependant pathway modulated by cytokines. Cancer Biol Ther 6:912-919

Dingli D, Michor F (2006) Successful therapy must eradicate cancer stem cells. Stem Cells 24:2603-2610

Faber E, Naušová J, Jarošová M, Egorin MJ, Holzerová M, Rožmanová S, Marešová I, Divoký V, Indrák K (2006) Intermittent dosage of imatinib mesylate in CML patients with a history of significant hematologic toxicity after standard dosing. Leuk Lymphoma 47:1082-1090

Frank SA, Iwasa Y, Nowak M (2003) Patterns of cell division and the risk of cancer. Genetics 163:1527-1532

Garner AL, Lau YY, Jordan DW et al. (2006) Implications of a simple mathematical model to cancer cell population dynamics. Cell Prolif 39:15-28

Hochhaus A, La Rosée P (2004) Imatinib therapy in chronic myelogenous leukemia: strategies to avoid and overcome resistance. Leukemia 18:1321-1331

Horn M, Loeffler M, Roeder I (2008) Mathematical modeling of genesis and treatment of chronic myeloid leukemia. Cells Tissues Organs 188:236-247

Ichimaru M, Ishimaru T, Mikami M, Yamada Y, Ohkita T (1981) Incidence of leukemia in a fixed cohort of atomic bomb survivors and controls, Hiroshima and Nagasaki, October 1950-December 1978. Technical Report no. 13-81. Hiroshima, Radiation Effects Research Foundation

Iwasa Y, Michor F, Nowak M (2004) Stochastic tunnels in evolutionary dynamics. Genetics 166:1571-1579

Jackson AL, Loeb LA (1998) The mutation rate and cancer. Genetics 148:1483-1490

Knudson AG (2001) Two genetic hits (more or less) to cancer. Nat Rev Cancer 1:157-170

Komarova NL, Wodarz D (2007) Stochastic modeling of cellular colonies with quiescence: an application to drug resistance in cancer. Theor Popul Biol 72:523-538
Loeb LA, Essigmann JM et al.(2002) Lethal mutagenesis of HIV with mutagenic nucleoside analogs. Proc Natl Acad Sci 96:1492-1497

Martinelli G, Soverini S, Iacobucci I, Baccarani M (2009) Intermittent targeting as a tool to minimize toxicity of tyrosine kinase inhibitor therapy. Nat Clin Pract Oncol 6:68-69

Michor F, Nowak M, Frank SA, Iwasa Y (2003) Stochastic elimination of cancer cells. Proc R Soc Lond B 270:2017-2024

Michor F, Hughes TP, Iwasa Y, Branford S, Shah NP, Sawyers CL, Nowak MA (2005) Dynamics of chronic myeloid leukemia. Nature 435:1267-1270

Michor F, Nowak M, Iwasa Y (2006) Evolution of resistance to cancer therapy. Curr Pharm Des 12:261-271

Michor F (2007) Chronic myeloid leukemia blast crisis arises from progenitors. Stem Cells 25:1114-1118

Moran PAP (1962) The statistical processes of evolutionary theory. Oxford: Clarendon Press

Müller MC, Lahaye T, Hochhaus A (2002) Resistance to tumor specific therapy with imatinib by clonal selection of mutated cells. Dtsch Med Wochenschr 127:2205-2207

Nowak MA, Komarova NL, Sengupta A, Jallepalli PV, Shih I, Vogelstein B, Lengauer C (2002) The role of chromosomal instability in tumor initiation. Proc Natl Acad Sci 99:16226-16231

Pizzolato N, Valenti D, Persano Adorno D et al. (2009) Evolutionary dynamics of imatinib-treated leukemic cells by stochastic approach. Cent Eur J Phys 7:541-548

Rea D, Etienne G, Corm S, Cony-Makhoul P, Gardembas M, Legros L, Dubruille V, Hayette S, Mahon F-X, Cayuela J-M, Nicolini FE (2009) Imatinib dose escalation for chronic phase-chronic myelogenous leukaemia patients in primary suboptimal response to imatinib $400 \mathrm{mg}$ daily standard therapy. Leukemia 23:1193-1196

Roeder I, Horn M, Glauche I, Hochhaus A, Mueller MC, Loeffler M (2006) Dynamic modeling of imatinib-treated chronic myeloid leukemia: functional insights and clinical implications. Nat Med 12:1181-1184

Sabnis GJ, Macedo LF, Goloubeva O, Schayowitz A, Brodie AMH (2008) Stopping treatment can reverse acquired resistance to letrozole. Cancer Res 68:4518-4524

Seruga B, Tannock IF (2008) Intermittent androgen blockade should be regarded as standard therapy in prostate cancer. Nat Clin Pract Oncol 5:574-576

Shah NP, Kantarjian HM, Kim D, Réa D, Dorlhiac-Llacer PE, Milone JH, Vela-Ojeda J, Silver RT, Khoury HJ, Charbonnier A, Khoroshko N, Paquette RL, Deininger M, Collins RH, Otero I, Hughes T, Bleickardt E, Strauss L, Francis S, Hochhaus A (2008) Intermittent target Inhibition with dasatinib $100 \mathrm{mg}$ once daily preserves efficacy and improves tolerability in imatinibresistant and -intolerant chronic-phase chronic myeloid leukemia. J Clin Oncol 26:3204-3212

Sokal JE, Baccarani M, Russo D, Tura S. (1988) Staging and prognosis in chronic myelogenous leukemia. Semin Hematol 25:49-61

Vigneri P, Wang JY (2001) Induction of apoptosis in chronic myelogenous leukemia cells throught nuclear entrapment of BCR-ABL tyrosine kinase. Nat Med 7:228-234

Weisberg E, Griffin JD (2001) Mechanisms of resistance imatinib (STI571) in preclinical models and in leukemia patients. Drugs Resist Updat 4:22-28

Weisberg E, Griffin JD (2003) Resistance to imatinib (Glivec): update on clinical mechanisms. Drugs Resist Updat 6:231-238

Zhdanov VP (2008) Stochastic model of the formation of cancer metastases via cancer stem cells. Eur Biophys J 37:1329-1334 